\title{
Explorando o DP: a presença da categoria NumP*
}

\author{
DP internal positions: a NumP category
}

\author{
Marina R.A. Augusto \\ UERJ/PUC-Rio/LAPAL \\ José Ferrari Neto \\ PUC-Rio/LAPAL \\ Letícia M. Sicuro Corrêa \\ PUC-Rio/LAPAL
}

\section{Abstract}

This paper focuses on NumP, an independent projection for number, which was proposed in pre-minimalist research (Picallo, 1991; Ritter, 1991). Arguments for the relevance of maintaining it as an independent projection in a minimalist framework, contrary to Chomsky (1999), who assumes number is one of the j-features associated to $\mathrm{N}$, are provided. The consequences such presence imposes for DP internal agreement phenomenon are discussed and some alternatives to account for it are offered.

Keywords

Minimalism, formal features, singular/plural.

\section{Resumo}

Este artigo trata da projeção independente NumP, proposta no arcabouço gerativista pré-minimalista (PICALLO, 1991; RITTER, 1991). Embora Chomsky (1999) assuma que número é um dos traços- $j$ associados a $\mathrm{N}$, argumenta-se pela relevância de se manter NumP no arcabouço minimalista e discutem-se as conseqüências que essa presença traz para 
a questão da concordância interna ao DP, apontando-se para algumas alternativas de análise.

\section{Palavras-chave}

Minimalismo, traços formais, singular/plural. 


\section{1- INTRODUÇÃO}

possibilidade de se assumirem projeções intermediárias entre o NP
e o DP se torna tema da literatura gerativista, desde que a proposta
de Abney (1987) passou a ser incorporada na teoria. A presença de categorias intermediárias é apontada seja para derivar relações de ordem e distribuição complementar (ou não) entre artigos, demonstrativos e possessivos (CINQUE, 1994; BRUGÈ, 2002; CERQUEIRA, 1996), seja para dar conta das relações de concordância entre os elementos do sintagma determinante (RITTER, 1991; PICALLO, 1991). Nesse domínio, Ritter (1991) propõe a existência de NumP entre D e N, com base em dados do hebraico, enquanto Picallo (1991) sugere que a presença de marcas de gênero e número seja um reflexo do movimento sucessivo cíclico do núcleo N. A autora propõe GenP, localizado entre o NP e NumP. Assim, a seqüência de afixos espelharia a hierarquia das categorias funcionais, assumindo-se que $\mathrm{N}$ se mova até o núcleo Num, passando por Gen.

No Programa Minimalista, no entanto, Chomsky (1995 e subseqüentes) tem assumido que número e gênero, conjuntamente com pessoa, compõem um conjunto de traços, denominados traços- $\varphi$, a serem associados a categorias lexicais ou funcionais no esqueleto sintático, e remete a dois mecanismos de concordância: via atuação de Agree, uma das operações do sistema computacional, e um mecanismo denominado Concord, que diria respeito à concordância interna ao DP, disparada via Merge tão somente (CHOMSKY, 1999). Desse modo, os traços- $\varphi$ são tomados como traços interpretáveis no nome, enquanto os determinantes apresentam traços- $\varphi$ não interpretáveis, cuja valoração se dá via Merge, sob atuação de Concord.

Raposo (1998) e Magalhães (2004) discordam dessa distribuição assumida em Chomsky. Para o primeiro autor, os traços interpretáveis de um argumento, isto é, os traços de número e de pessoa, devem ser associados a D e não a N. Magalhães (2004) assume que o determinante possui traços de 
número interpretável e de gênero não-interpretável, enquanto o nome possui traços de número não-interpretável e de gênero e pessoa interpretáveis. Adotando essa distribuição, Magalhães (2004) defende que a relação independente Concord, proposta para dar conta da concordância interna ao DP, é desnecessária. Segundo ela, pode-se lançar mão da operação Agree, tomando-se como base a proposta do próprio Chomsky (1999) para a valoração dos traços formais de construções participiais.

Este artigo tem por objetivo explorar a possibilidade de se adotar uma projeção NumP no domínio nominal, uma vez que número pode ser tomado como um traço que não é necesariamente interpretável em $\mathrm{N}$, e verificar as implicações que derivam dessa presença para o mecanismo de concordância interna ao DP.

Na seção seguinte, expõe-se o mecanismo de concordância adotado no PM e a discussão levantada por Magalhães (2004) acerca da possibilidade de se estender o mecanismo Agree para a concordância interna ao DP, com base na proposta de Chomsky para as construções participiais. A Seção 3 traz argumentos a favor de se considerar que número projeta uma categoria funcional independente. Dados do português (e do espanhol) que parecem apontar para a relevância de não se considerar que número seja interpretável em N são apresentados na Seção 4. As seções seguintes exploram as implicações decorrentes da presença de NumP no esqueleto estrutural, seja em termos da possibilidade de se associarem parâmetros à questão do número (MUNN \& SCHMITT, 2001; DEPREZ, 2005), seja em relação às assunções que se farão necessárias para a questão da concordância interna ao DP. A conclusão do artigo, na seção final, favorece a hipótese de que NumP constitui uma projeção independente no DP.

\section{2- CONCORD, AGREE E CONSTRUÇÕES PARTICIPIAIS}

Em Chomsky (1999), o autor remete a dois mecanismos de concordância: via atuação de Agree, e um mecanismo denominado Concord, a que ele se refere em nota e diria respeito à concordância interna ao DP, disparada via Merge tão somente.

A presença de traços interpretáveis e não-interpretáveis de mesma dimensão associados a categorias distintas expressa o mecanismo de concordância sintática presente nas línguas naturais. Traços não-interpretáveis 
devem ser eliminados na sintaxe. A operação Agree é responsável por deflagrar a busca por traços de mesma dimensão a serem pareados, possibilitando a eliminação dos traços não-interpretáveis. Em Chomsky (1999), essa idéia é implementada assumindo-se que os traços não-interpretáveis são traços sem valor especificado que devem ser valorados no decorrer da derivação sintática. Assim, o conjunto de traços não-interpretáveis, denominado sonda, procura um conjunto de traços similar - o alvo - para que Agree se estabeleça e os traços nãointerpretáveis, uma vez valorados, sejam eliminados para LF, embora essa valoração possa produzir efeitos visíveis em PF. Concomitantemente à atuação de Agree, a operação Move, entendida na verdade como Copy + Merge, efetivase quando uma posição de especificador é disponibilizada pela sonda - dada a presença de um traço do tipo EPP. O traço EPP é, nessa versão do modelo, tomado como um traço selecional que é satisfeito pela concatenação de determinado elemento no especificador da categoria ao qual está associado. Assim, é a presença do traço EPP que determinará se Merge (interno) será acionado após a operação Agree ter valorado o(s) traço(s) não-interpretável(is) do alvo, isto é, se Move atuará. É também relevante mencionar em relação ao mecanismo de valoração de traços não-interpretáveis que estes só são valorados se combinados com um elemento que apresente um conjunto completo de traços- $\varphi$. Isso significa que elementos defectivos são permitidos no sistema, o que pode levar a que a operação Agree seja deflagrada mais de uma vez para um determinado conjunto de traços não-interpretáveis.

Em relação à atuação de Concord, Chomsky admite-a no caso da concordância interna ao DP, ao considerar que os traços- $\varphi$ são interpretáveis nos nomes, sendo o traço de número opcional e o de gênero intrínseco, enquanto os determinantes apresentariam traços- $\varphi$ não interpretáveis, cuja valoração se daria via Merge, sob atuação de Concord.

Magalhães (2004) questiona a necessidade da relação independente Concord para dar conta da concordância interna ao DP. Segundo ela, podese lançar mão da operação Agree, tomando-se como base a proposta do próprio Chomsky (1999) para a valoração dos traços formais de construções participiais. Para tanto, a autora assume que o determinante (D) possui traços de número interpretável e de gênero não-interpretável, enquanto o nome (N) possui traços de número não-interpretável e de gênero e pessoa interpretáveis. 
No sistema de valoração dos traços formais de construções participiais, Chomsky assume que o particípio apresenta traços de gênero, número e Caso não-interpretáveis. $\mathrm{O}$ autor demonstra como os traços de Caso do particípio e do nominal serão valorados a depender de uma sonda mais alta, a partir dos seguintes exemplos [(18) (a) e (b) no original)]:

(1)
(a) $\left[_{C}\left[{ }_{\beta} T\right.\right.$ seem

[EXPL to have been $\left[{ }_{\alpha}\right.$ caught [several fish]]]]

(b) $\left[_{\beta} \mathrm{V}\right.$ expect

No domínio $\alpha$, o particípio valora seus traços de gênero e número a partir dos valores interpretáveis desses traços no nominal, mas os traços de Caso dos dois elementos continuam sem valor, o que permite que ambos elementos permaneçam disponíveis para entrar em relações subseqüentes de concordância.

(2)

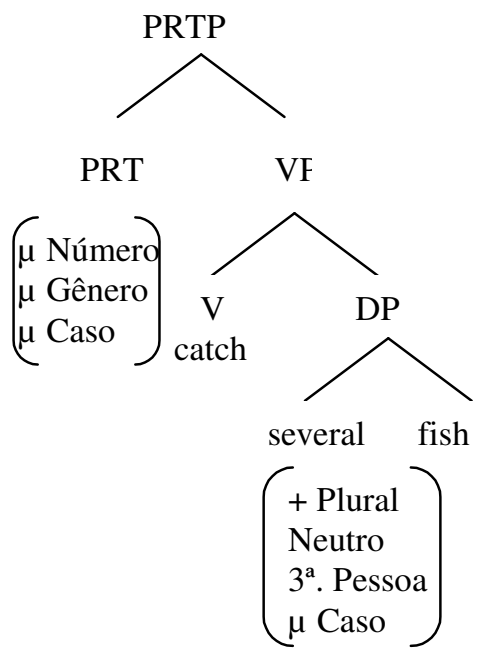

A camada do infinitivo constitui tempo defectivo, impossibilitado de valorar um traço de Caso, cujo requerimento de especificador preenchido traço EPP - é satisfeito pela concatenação do expletivo there. Sendo assim, o particípio e o nominal continuam sem ter seu traço de Caso valorado. 
(3)

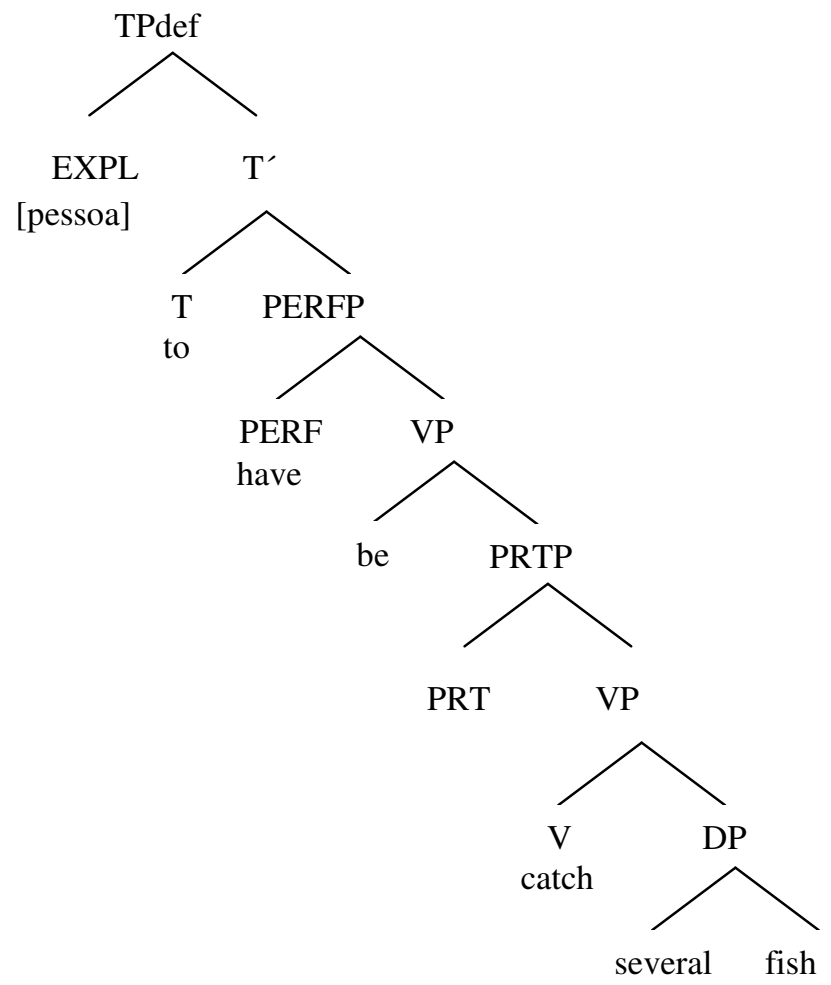

A valoração do traço de Caso se dará, portanto, a partir de uma sonda superior, no domínio $\beta$, a depender da construção.

Na presença do verbo to expect, vP está presente e a valoração se dá em cascata, na busca pelos traços- $\varphi$, sendo que os elementos são defectivos, não possuem um conjunto completo de traços- $\varphi,-$ o expletivo apresenta somente o traço de pessoa e o partícipio apresenta traços de gênero e número - permitindo assim que a sonda continue sua busca até o nominal. Os traços de Caso serão valorados como acusativo, uma vez que os elementos entraram em uma relação de checagem $\operatorname{com} v$.

No caso do verbo to seem, um verbo inacusativo, a camada vP não está presente (ou é defectiva). O EPP e os traços- $\varphi$ de T acionam a busca por um alvo. Da mesma maneira, haverá uma valoração em cascata. $O$ expletivo satisfará, ainda, o traço EPP do T de seem. Os elementos recebem caso nominativo. 
(4)
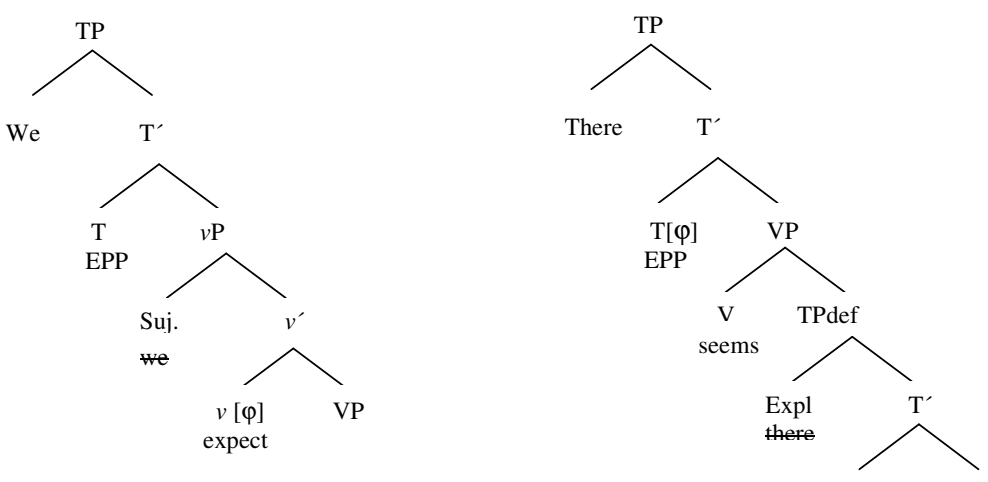

TPdef

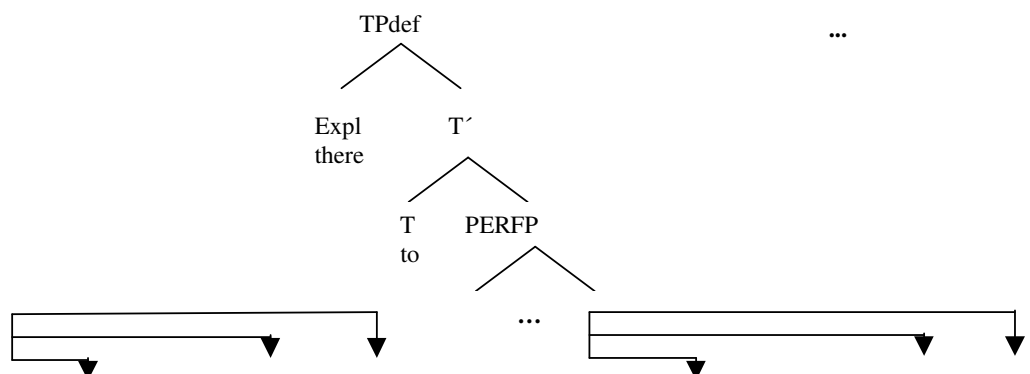

$\cdots$

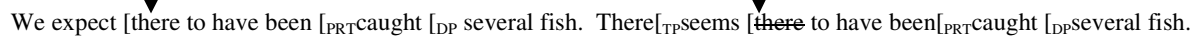
Acus.

Nom.

A implementação da valoração de traços em cascata é estendida por Magalhães para o domínio DP. Conforme mencionado, a autora assume, no entanto, diferentemente de Chomsky, que o traço de número é não-interpretável em N, sendo interpretável em D [conforme Olsen (1989) e Longobardi (1994)]. 
(5)

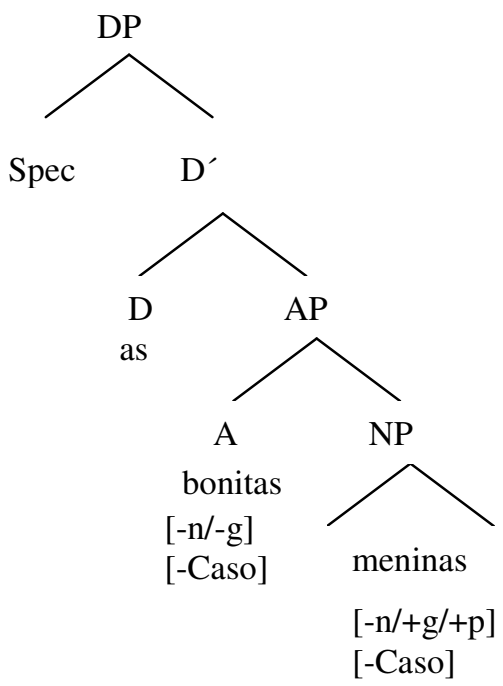

Nessa estrutura, uma primeira relação de concordância se estabelece entre o adjetivo e o nome, resultando na valoração do traço de gênero do adjetivo. Outra relação de concordância é disparada pelo traço nãointerpretável de D. O adjetivo é encontrado, o traço de número do adjetivo é valorado, mas o traço de gênero do adjetivo é não-interpretável, não podendo, portanto, valorar o traço de $\mathrm{D}$, que segue em busca de um traço de gênero interpretável, encontrando-o em N.

É mister observar que Magalhães assume que o traço de gênero presente no adjetivo, embora já valorado, não valoraria o traço de gênero de $\mathrm{D}$, que continuaria a busca até $\mathrm{N}$, onde esse traço é interpretável. A análise não pode manter a assunção em Chomsky (1999) de que somente um conjunto de traços- $\varphi$ completo seria capaz de valorar traços não-interpretáveis. Se, por um lado, poderia se verificar essa imposição no que concerne à busca de $\mathrm{D}$ pelo traço de gênero, que passa pelo adjetivo e segue até $\mathrm{N}$, que contém traços de número, gênero e pessoa, por outro lado, o próprio $\mathrm{D}$, portador de traços de número e gênero (sem pessoa), é capaz de valorar o traço de número do adjetivo e, posteriormente, de N. Vale lembrar que Raposo (1998), diferentemente de Chomsky (1999) e Magalhães (2004), defende que o traço de pessoa deve ser interpretável em $\mathrm{D}$ e não em $\mathrm{N}$. Voltaremos a essas questões de implementação das análises adiante. 
Após a valoração dos elementos internos ao DP, esse vai se combinar com uma sonda, T ou v, e valorar o seu traço de Caso também em cascata. Nessa relação, é a busca pelo traço de pessoa que permitirá a valoração em cascata, de maneira equivalente ao sugerido para as construções participiais.

Em suma, a análise de Chomsky (1999) associa o traço de número a $\mathrm{N}$ e a concordância interna ao DP se faz pelo mecanismo Concord, amparado em Merge tão somente. Em Magalhães (2004), questiona-se a presença de número interpretável em $\mathrm{N}$ e busca-se uma análise para a concordância interna ao DP com base na operação Agree, remetendo-se à análise chomskyana para os particípios. No entanto, essa distribuição de traços- $\varphi$ implica abandonar a exigência de que somente um conjunto de traços- $\varphi$ completo possa valorar suas contrapartes não-interpretáveis.

Neste artigo, aponta-se para uma outra possibilidade: a de que número seja projetado independentemente. Além disso, verificam-se as implicações que derivam dessa presença da categoria independente NumP no DP. A possibilidade de dar conta da concordância interna ao DP em cascata, conforme sugerido por Magalhães (2004), poderia ser estendida a uma estrutura que incorporasse projeções do tipo NumP (ou mesmo GenP)? Relações pertinentes às interfaces lingüísticas sustentam uma análise em que um parser lingüisticamente informado analisa membros de classes fechadas como núcleos funcionais. Desse modo, o estabelecimento da distinção entre traços opcionais e intrínsecos poderia se dar a partir do processamento da concordância no âmbito do DP: traços opcionais seriam projetados como categorias funcionais (GenP, NumPe D, ou outra), uma vez que a criança identificasse informação pertinente a eles em PF (na fonologia/morfologia) (CORRÊA, AUGUSTO \& FERRARI-NETO, 2005). ${ }^{1}$ A discussão acerca da relevância dessas distinções para a proposta da categoria independente NumP, além de argumentos adicionais favoráveis à presença de NumP no DP são apresentados na próxima seção. Alternativas para derivar a concordância interna ao DP, composto de uma projeção independente NumP, serão discutidas mais adiante.

\section{3- ARGUMENTOS PRÓ-NumP}

As propostas acerca de uma projeção independente relativa a número apóiam-se em diferentes argumentos. Um aspecto morfológico apontado como evidência para a projeção independente NumP diz respeito à existência 
de morfemas livres de número em línguas como Tagalog (falada nas Filipinas) ou Yapese (falada na Micronésia). Os exemplos a seguir são do Yapese (DRYER, 1989):

$\begin{array}{ccc}\text { a. ea rea } & \text { kaarroo } & \text { neey } \\ \text { sing } & \text { carro } & \text { este } \\ \text { b. ea gal } & \text { kaarroo } & \text { neey } \\ \text { dual } & \text { carro } & \text { este } \\ \text { c. ea pi } & \text { kaarroo } & \text { neey } \\ \text { plural } & \text { carro } & \text { este }\end{array}$

A necessidade de uma projeção intermediária entre NP e DP para derivar questões de ordem também tem levado a se sugerir que essa projeção poderia ser NumP. Essa discussão é levantada em relação à ordem nome e adjetivo, por exemplo. $\mathrm{O}$ adjetivo está para essa questão assim como o advérbio esteve para a discussão acerca da ordem entre verbo e advérbio, no nível do IP. Pollock (1989), comparando o inglês e o francês, propôs que a ordem básica seria advérbio e verbo e a ordem exibida pelo francês (verbo/ advérbio) seria resultante do movimento do verbo para uma projeção funcional mais alta (TP ou AgrP). A comparação com adjetivos, no nível do sintagma nominal, impõe-se. As línguas românicas, por exemplo, tendem a apresentar a ordem verbo/advérbio assim como nome/adjetivo. Defendendose que a ordem básica (CINQUE, 1994) seria adjetivo e nome (conforme a ordem predominante nas línguas germânicas), chega-se ao raciocínio, em paralelo ao defendido para o nível da sentença, de que a ordem nome/ adjetivo, encontrada nas línguas românicas, seria resultado de movimento do nome para uma projeção funcional entre DP e NP.

Há também ponderações de ordem semântica em relação ao papel desempenhado por uma projeção funcional dedicada ao número. Sauerland (2003) afirma ter sido a pesquisa acerca da semântica do número fortemente influenciada pela distribuição de marcas morfológicas explícitas de número. Bennett (1974) foi um dos primeiros a assumir que a marca de plural nos nomes deveria ser de alguma maneira interpretável, enquanto esse traço no verbo seria não-interpretável, um puro reflexo de concordância sintática com o sujeito. Sauerland observa que, no alemão, a marca morfológica explícita de plural aparece também no determinante, o que indica que haveria outras 
opções a considerar em relação ao local de interpretabilidade semântica desse traço. Sua proposta, no entanto, é de que o número nuclearia sua própria projeção, localizada acima do DP, sendo que os traços de número seriam semanticamente interpretados aí unicamente. A análise de Sauerland explicita formalmente a possibilidade de dois DPs singulares, uma vez coordenados, terem uma interpretação de plural, informação relevante seja para a concordância com o verbo, quando estes elementos estão em posição de sujeito, seja para a retomada por um elemento co-referencial plural.

Apontando para relações sintáticas e semânticas, Vangsnes (2001) menciona que já Abney (1987) propõe uma projeção QP (Quantifier Phrase) entre DP e NP. A distinção, no entanto, entre quantificadores existenciais e universais levou à proposta de que os últimos estariam abrigados sob uma projeção acima de DP (SHLONSKY, 1991; GIUSTI, 1991). Vangsnes sugere que a afinidade entre quantificadores existenciais e os numerais justificaria uma identificação entre esse QP mais baixo e a projeção NumP, sugerida por Ritter (1991), em associação à morfologia de número. Para Vangsnes, a presença de NumP está relacionada à cardinalidade/contagem (countability), distinguindo entre leituras massivas e contáveis do nome a que se associa. Vangsnes deriva as propriedades referenciais de um DP a partir da presença de categorias funcionais no domínio nominal. As categorias substantivas/ lexicais contribuem a intensão (sentido) do termo, enquanto as categorias funcionais fornecem uma âncora extensional para o termo, isto é, a referência. A operação desempenhada por número remete à definição do tipo de conjunto (átomos ou pluralidades) sendo tomado.

Borer (2005) admite duas projeções universais intermediárias entre DP e NP - $\mathrm{CL}_{\max }$ e \#P. Para essa autora, todos os nomes são massivos e precisam ser parcelados (portioned out) - camada CL (a dividing head), antes de interagirem com um sistema de contagem (count system) - camada \#P. $\mathrm{CL}_{\max }$ é responsável pela geração da distinção massivo vs. contável, enquanto \#P é o local para a definição específica de quantidades.

Outro argumento que pode favorecer a presença de uma categoria independente dedicada a número é de natureza formal e diz respeito à arquitetura defendida por Chomsky (1995 e subseqüentes) para caracterizar o sistema computacional. O Minimalismo é parcimonioso em relação à presença de projeções funcionais. Estas só se justificam se possuem importe semântico. Sendo assim, a presença de NumP, em paralelo a TP, sustenta-se 
uma vez que a noção de número constitui um traço semanticamente interpretável, assim como a presença da noção de tempo (interpretável) justifica T. Ainda, em relação à arquitetura do sistema computacional e suas relações com as interfaces lingüísticas, seria interessante verificar em que medida não se pode relacionar o caráter intrínseco ou opcional dos traços à possibilidade de projeções independentes. Os traços de caráter intrínseco estariam associados a itens lexicais, fazendo parte da sua entrada lexical juntamente com traços de natureza semântica ou fonológica, enquanto os traços de caráter opcional fariam parte do léxico da língua em questão, mas não estariam diretamente associados a itens específicos, sendo buscados no momento de formar a Numeração, constituindo informação sintática independente que nuclearia uma projeção independente. No caso de número, novamente, a semelhança com Tempo se apresenta. Nesse sentido, traços formais com importe semântico pertinente à referência constituiriam núcleos que projetariam categorias funcionais independentes. Essa propriedade teria a vantagem de facilitar a incorporação de operações computacionais em modelos de processamento (cf. CORRÊA \& AUGUSTO, 2005), o que é relevante para uma teoria de língua que se apresenta como cognitiva.

Em suma, a partir dos argumentos apresentados, parece plausível defender-se a presença de uma projeção funcional NumP na estrutura do DP.

\section{4- DADOS DO PORTUGUÊS: AQUISIÇÃO DE NÚMERO E ELIPSE NOMINAL}

Em relação aos dados do português particularmente, abordaremos algumas questões relacionadas à aquisição de número e enfocaremos características do funcionamento da elipse nominal que, embora não sejam decisivos no que concerne a evidenciar a presença de NumP, se mostram incompatíveis ora com a idéia de que número seja interpretável em $\mathrm{N}$, ora em D.

\subsection{Aquisição de número}

Os resultados obtidos acerca da percepção/interpretação da marca de número no DP, pelo GPPAL-LAPAL, ${ }^{2}$ não permitem decidir acerca da presença (ou não) de uma projeção independente NumP. A criança deve identificar informação relativa a número gramatical a partir da informação 
morfofonológica e da ordenação dos elementos no fluxo da fala, para os quais se atribui uma interpretação semântica particular. Assumindo-se um modelo de língua a partir de uma orientação minimalista, a informação sintática de número relaciona-se ou a um traço associado ao nome (ou ao $\mathrm{D}$, segundo Magalhães) ou à projeção independente NumP e se expressa por marcas morfológicas distintas entre as línguas.

No caso do português, o plural é marcado pela presença de um morfema $-s$. Esse morfema pode aparecer em vários elementos no DP determinantes, adjetivos e nomes - caracterizando o dialeto padrão, sendo, no entanto, que um outro sistema de marcação coexiste com esse: o nãopadrão em que a informação de número tende a ser visível na interface fonética apenas no Determinante (ou no elemento mais à esquerda no DP). Assume-se que a relação entre os elementos do DP em termos de valor para número se dá segundo a visão de concordância sintática já discutida anteriormente, ou seja, pela noção de que traços interpretáveis e nãointerpretáveis são pareados e o valor do traço interpretável é assumido para os elementos que apresentam traços não-interpretáveis de número. Se essa relação é mediada por meio da projeção NumP ou não é difícil determinar, para o PB, a partir dos dados da língua. No entanto, em relação ao modo como a criança extrai informação do input que recebe, Corrêa (2002), Ferrari-Neto (2003) e Ferrari-Neto, Corrêa \& Augusto (2005) assumem que o processamento da concordância é instrumental para a identificação de propriedades específicas dos traços formais da língua em aquisição, tendo sido verificado em Corrêa, Augusto, Ferrari-Neto \& Name (2004) que a manipulação de marcas incongruentes de número no DP, isto é, entre o determinante e o nome, evidenciava que a informação proveniente da flexão do nome era predominantemente levada em conta pelas crianças para o estabelecimento da referência. Lopes (2004) aponta que emissões iniciais das crianças contemplam nomes com a marca do morfema de plural, sem manifestação desse morfema no determinante, o que não é viável na língua e não faria parte do input a que a criança está exposta.
a. a hienas (C. 2;4)
b. meu chinelinhos
(C. 2;6)

Por outro lado, Corrêa, Augusto \& Ferrari-Neto (2005) apresentam um quadro em que as crianças se mostram sensíveis às possibilidades gramaticais 
na língua. Nesse experimento, utilizam-se figuras de objetos inventados e pseudonomes para se referir aos objetos. O propósito é verificar se as crianças com idade média de 24 meses são capazes de identificar um referente com base no processamento da concordância de número no DP. Pede-se à criança que mostre algo a um fantoche, nomeado Dedé, a partir de um álbum de figuras no qual cada página contém quatro desenhos: três de objetos desconhecidos, dois deles de objetos unitários e o terceiro apresentando várias instâncias de um mesmo objeto. Manipulam-se as incongruências entre determinante e nome, em relação à marcação de número, levando-se em conta as duas variantes do PB como realizações gramaticais - forma padrão e não-padrão - e dois tipos de expressão morfológica de número não gramaticais no $\mathrm{PB}$ - número como sufixo exclusivo no nome e como infixo - utilizando-se a forma gramatical do singular como controle.

A seguir, a exemplificação das condições e a ilustração de uma das pranchas que compõem o álbum:

\section{> Plural Gramatical:}

Mostra os dabos pro Dedé. (Padrão)

Mostra os dabo pro Dedé. (Não-padrão)

> Plural Agramatical:

Mostra o dabos pro Dedé. (sufixo em $\mathbf{N}$ ) Mostra o dasbo pro Dedé. ( infixo em N)

$>$ Controle Singular:

Mostra o dabo pro Dedé.
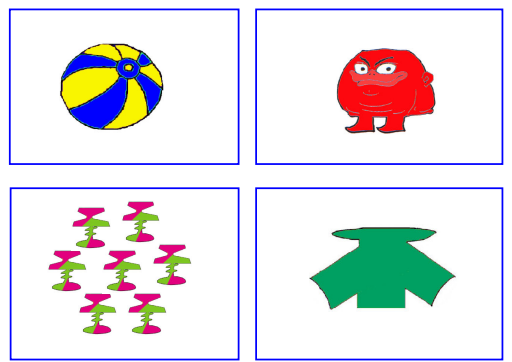

Os resultados obtidos apontam para um maior número de respostas da figura múltipla para as condições gramaticais $(\mathrm{t}(17)=5.65 \mathrm{p}<.0001)$ (Gráfico 1), não havendo diferença significativa entre o plural padrão ou o nãopadrão $(\mathrm{t}(17)=1.16 \mathrm{p}=.26)$ (Gráfico 2). As formas agramaticais também apresentam percentuais de resposta bastante similares, não havendo diferença significativa entre as condições com sufixo exclusivo no nome ou infixo em $\mathrm{N}(\mathrm{t}(17)=0.97 \mathrm{p}=.34)($ Gráfico 2): 
(9)

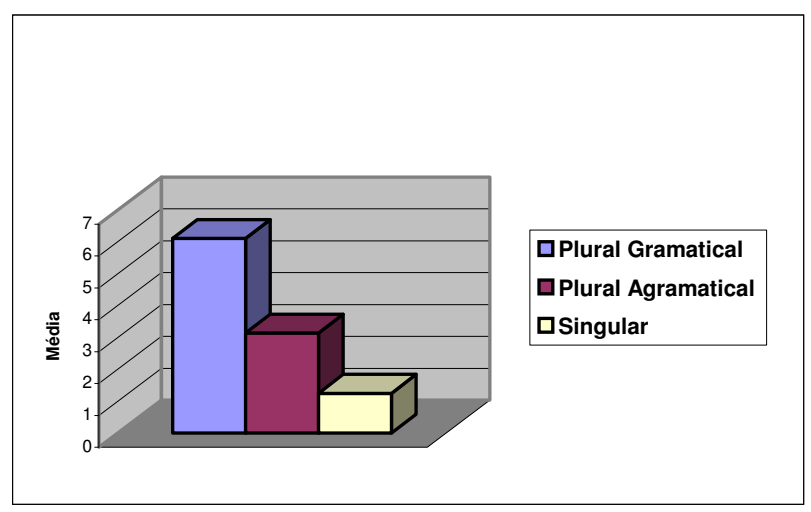

GRÁFICO 1: Média de respostas - figura múltipla

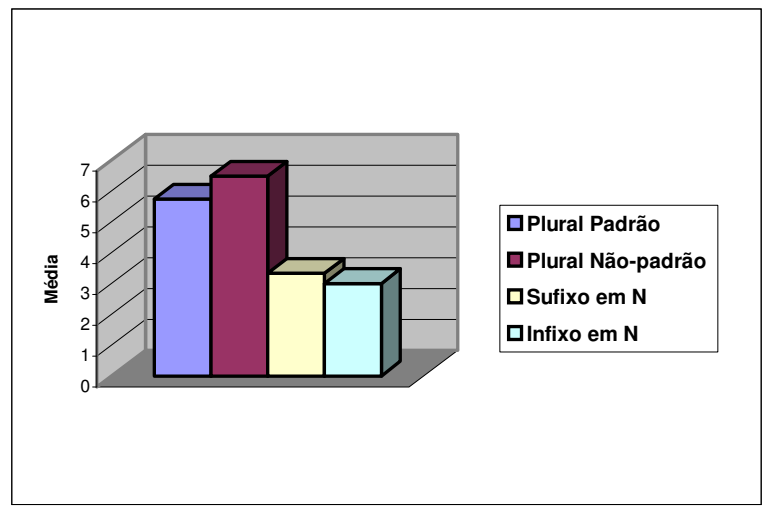

GRÁFICO 2: Média de respostas - figura múltipla em função da presença de -s

A tarefa permitiu verificar que as crianças são capazes de identificar corretamente um conjunto de objetos desconhecidos levando em conta a informação de plural no DP, seja em que variante da língua for, padrão ou nãopadrão, o que sugere que essas crianças reconhecem número como um traço formal, identificam os valores associados a esse traço, particularmente a forma marcada plural e apóiam-se no mecanismo de concordância a fim de adquirir nomes novos, conforme será explicitado adiante. 
Esses resultados indicam que as crianças identificam informação morfológica relativa a número $\mathrm{em} \mathrm{D}$, independentemente do dialeto a que estão predominantemente expostas. $\mathrm{O}$ que permitiria à criança atribuir ao $\mathrm{DP} \mathrm{o}$ número identificado em $\mathrm{D}$ ? Consideramos que o pressuposto de haver concordância no interior do DP, sendo o morfema de número em D reflexo dela, pode explicar a atribuição do número identificado em $\mathrm{D}$ ao $\mathrm{DP}$, visto que o morfema de número em $\mathrm{D}$ seria a expressão do resultado da concordância em PF. Alternativamente, pode-se considerar (como o faz Magalhães, 2004) que o traço de número é interpretável em $\mathrm{D}$, o que não implicaria processamento da concordância interna ao DP por parte da criança. Número pode ser tomado como um traço semântico. Contudo, o fato de dados da produção como os relatados por Lopes (2004) (ver (7)) sugerirem uma indefinição por parte da criança com relação à identificação do modo como o traço de número se expressa em PF pode ser visto como indicativo de que o local da interpretabilidade do traço de número no DP não é uma questão passível de ser decidida em função do local de onde essa informação é extraída na morfologia. Sendo assim, considera-se que a criança precisa identificar número como um traço formal da língua, o qual pode nuclear uma projeção independente do tipo NumP, assim como as possíveis realizações morfológicas associadas a esse traço. ${ }^{3}$ Nesse sentido, prevê-se que, quanto maior a variabilidade na expressão morfológica de número em uma língua, maior a instabilidade nos dados advindos da produção espontânea de crianças na aquisição da língua.

Assumindo-se NumP, Corrêa, Augusto \& Ferrari-Neto (2005; 2006) sugerem que os seguintes passos estariam envolvidos na tarefa de compreensão de nomes novos marcados para o plural, conforme explorou o experimento relatado (adotando-se o DP como uma janela de processamento): 


$$
\text { PF: }\left[{ }^{\Omega} \mathrm{u} \int^{\prime} \operatorname{dabu}(\mathcal{}()]=O s \operatorname{dabo}(s)\right.
$$

Segmentação da fala

em unidades prosódicas

Reconhecimento de lexemas

Parsing 1: análise morfológica / de ordem de palavras e acesso aos lemas

$$
\left.\rightarrow \quad\left[{ }^{\Omega}\left[{ }^{\omega} \mathbf{u}\right]\right]\left[{ }^{\omega}, \mathbf{d a b u}\left(\int\right)\right]\right]
$$

$\rightarrow \quad$ /us/ /'dabo(s)/

$$
\text { ?! }
$$$$
\left.\rightarrow \quad\left[{ }_{\mathbf{F P}}[\mathbf{o}]_{\mathrm{f}}[\mathbf{s}]\right]_{\mathrm{f}}[\sqrt{ } \mathbf{?}(\mathbf{s})]_{l}\right]
$$
afixo ?! de número

$$
\left(\begin{array}{c}
\mathbf{D} \\
\text { Masc } \\
\text { Def }
\end{array}\right)\left(\begin{array}{c}
\text { Num } \\
\mathbf{P l}
\end{array}\right)\left(\begin{array}{l}
\mathbf{N} \\
?
\end{array}\right)
$$

Parsing 2: Computação sintática $\rightarrow$ (Merge e Agree assumindo-se LCA)

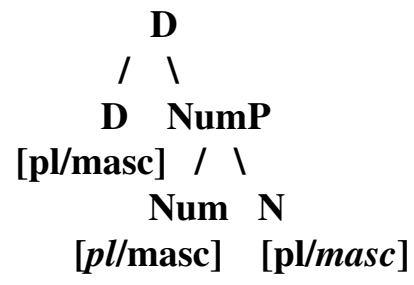

D

D NumP

Num $N$

$[p l /$ masc $] \quad[\mathrm{pl} / \mathrm{masc}]$

Interpretação semântica

$$
\rightarrow \quad \exists y[y=\sigma x * D x]
$$

Existe um $y$ tal que $y$ é o supremo do reticulado de todos os dabos e suas somas ${ }^{4}$

Representação conceptual

$\rightarrow \quad$ "Mais de uma entidade"

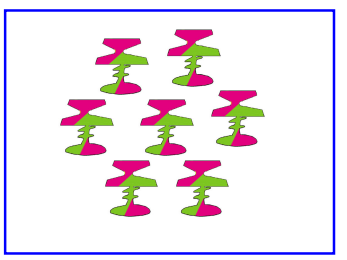

Referência
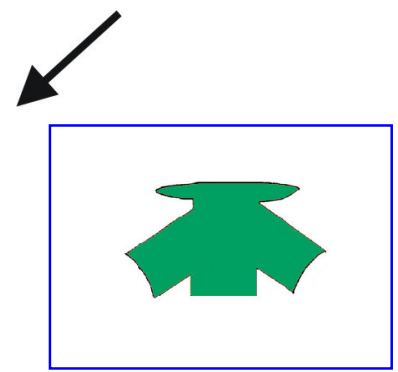
Essa visão atribui às propriedades “visíveis" para a criança nas interfaces um papel crucial na aquisição, constituindo-se em informação necessária para que ela identifique as propriedades específicas de sua língua, fazendo uso do acionamento de um sistema computacional disponível na espécie humana. Vale lembrar que, nessa conjugação entre modelo de língua e um mecanismo de processamento lingüístico instruído pela gramática universal, a noção de traços é de grande relevância para a aquisição da linguagem. A fim de que o sistema computacional seja acionado de maneira idêntica a do adulto, faz-se necessário que a correta identificação e associação dos traços relevantes a determinadas categorias possa ser realizada pela criança (AUGUSTO, 2003; 2006).

\subsection{Elipse nominal}

Um outro dado relevante para a discussão acerca do local do traço de número interpretável advém do fenômeno da elipse. Os dados a seguir contrastam elipse nominal com contraste de número e de gênero:

(11) a. O Pedro e o João são atores e o Paulo também é.

b. *Aquele senhor é barão e aquela senhora também é.

A associação da interpretabilidade do traço de número a uma categoria distinta de $\mathrm{N}$ parece ser adequada: a incongruência entre os traços de número de um antecedente e do material elidido resulta gramatical, mas incongruência de gênero é inaceitável (conforme aponta Zocca (2003), (ex. 11b) em relação ao predicativo no português, com algumas gradações relevantes).

Masullo \& Depiante (2004) e Saab (2005) também discutem esse fenômeno em relação ao espanhol, apontando para o seguinte contraste:

(12) a. Juan visitó a su tío y María visitó a los suyos.

b. *Juan visitó a su tío y María visitó a la suya.

Esses dados parecem favorecer uma análise em que os traços de gênero e número não estejam ambos associados a N. A adoção de NumP e o fato de que a elipse nominal alcança NP garantem que o número fique fora desse domínio, ou seja, os traços de número não ficam sujeitos à condição de identidade, diferentemente dos traços de gênero que, por estarem associados a $\mathrm{N}$, devem ser idênticos aos do antecedente na elipse. ${ }^{5}$ 


\section{5- NumP: ASSOCIAÇÃO A PARÂMETROS}

A adoção de NumP é assumida em propostas que associam a presença dessa projeção a parâmetros sintáticos. Nesse sentido, remetemos às propostas de Munn \& Schmitt (2001) e de Deprez (2005).

Os primeiros autores propõem a aplicação do Free Agr Parameter (BOBALJIK, 1995) ao domínio nominal. Línguas que possuem Agr e Num em projeções distintas (não fundidos) podem, opcionalmente, ter Num ausente. Segundo os autores, a possibilidade do singular bare em PB seria resultado da presença de um D nulo (RAPOSO, 1998) e da ausência de NumP, ou seja, o núcleo de número seria opcional no PB.

(13) In: Schmitt \& Munn (2000, p. 30)

a.

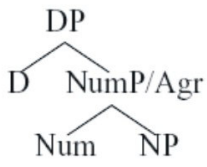

English b.

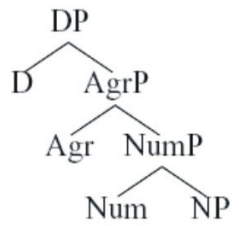

Romance

Deprez (2005) propõe um parâmetro morfológico que controla a presença sintática da projeção funcional para número. A presença de NumP força a realização de uma espécie (kind) em ocorrências, ou seja, é proposta uma relação entre a estrutura morfológica dos nominais bare e sua interpretação, dispensando-se a necessidade de uma parametrização semântica (CHIERCHIA, 1998).

(14) In: Deprez (2005, p. 867)

Plural Parameter

In + PL languages, NumP must systematically project, for count nouns at least - that is, even when the noun phrase is singular with no apparent overt "plural" or "singular" morphology — and this NumP must contain a counter, i.e., a measure function, which I take, as a first approximation, to be the translation of countabilty.

In_PL languages, on the other hand, the projection of NumP is optional and when it occurs, NumP does not have to contain a counter. ${ }^{6}$ 
Em relação ao fenômeno do bare singular no $\mathrm{PB}$, tal parâmetro parece poder ser estendido a essa língua, ao se assumir que os nomes comuns tenham uma denotação massiva, conforme sugerido por Muller (2002). No entanto, Schmitt \& Munn (2000) apresentam argumentos contra essa interpretação.

No que diz respeito à presença do bare singular na posição de sujeito em PB, observa-se que há concordância entre sujeito e verbo no singular, embora os nominais singulares bare possam ser antecedentes de pronomes no singular ou no plural (exemplos em Munn \& Schmitt, 2005, p. 825):

(15) a. Coelho vê o mundo de outra maneira porque é muito pequeno.

b. Coelho vê o mundo de outra maneira porque eles são muito pequenos.

Embora oferecer uma análise para os singulares bare não seja nosso objetivo aqui, é relevante apontar algumas especulações iniciais. Os fatos descritos em relação ao PB sugerem que os singulares bare sejam subespecificados para número. $\mathrm{O}$ comportamento desses nominais é distinto do comportamento de termos massivos. Sendo assim, parece adequado assumir que os massivos não apresentariam a camada NumP, mas que esta estaria presente nos nominais bare: se marcada para plural (e na presença de um D nulo), plurais bare seriam gerados; se não-especificada para número, os singulares bare seriam projetados. A concordância local com o sujeito assumiria o número default, ou seja, o singular. Já a retomada por pronomes leva em consideração a interpretação semântica gerada a partir da estrutura sintática, o que explica o uso de pronomes plurais. ${ }^{7}$

Levando-se em conta o parâmetro proposto por Deprez (2005), poderse-ia admitir uma parametrização mais fina, sugerida pela própria autora em nota, em que, além da variação atribuída à projeção NumP especificamente, a associação da obrigatoriedade do contador - "counter" - a essa projeção também admitisse variação. Nesse sentido, não seria necessário supor que o PB estivesse se tornando uma língua_PL, em que NumP fosse opcional, que parece ser uma caracterização muito mais adequada a línguas do tipo do chinês, mas a mudança poderia se dar na não-obrigatoriedade da associação do contador a NumP, o que explicaria o singular bare nessa variedade do português.

Em suma, as discussões anteriores mostram que freqüentemente se remete a uma projeção do tipo NumP seja em análises que se debruçam sobre a distinção massivo $v s$. contável, seja para a caracterização da distribuição de 
nominais bare nas diversas línguas. Na próxima seção, parte-se para as implicações que a presença dessa categoria traz no que diz respeito ao mecanismo de concordância sintática no âmbito interno do DP.

\section{6- NumP: QUESTÕES RELATIVAS À CONCORDÂNCIA INTERNA AO DP}

As análises que propuseram NumP (ou o assumiram) o fizeram dentro do arcabouço anterior ao minimalismo ou não se detiveram especificamente sobre a questão da concordância interna ao DP. Nesse sentido, há que se fazer uma reavaliação em relação ao mecanismo de checagem/valoração dos traços no DP em uma perspectiva minimalista, tendo em vista a discussão já adiantada na Seção 2.

Há três possibilidades que se apresentam: (i) manter o mecanismo adicional Concord, conforme defendido por Chomsky (1999), (ii) aproximar Num de T, no sentido de que se trata de uma operação semântica que atribui referência a elementos substantivos ( $\mathrm{N}$ ou $\mathrm{V}$ ), e cujo estabelecimento da relação é mediada via núcleos, ou (iii) manter a noção de que os traços- $\varphi$ são compartilhados pelos elementos presentes no DP e adotar Agree.

A primeira alternativa seria uma adaptação do sugerido por Chomsky (1999), assumindo-se a presença da categoria NumP, em que a valoração dos traços se daria via Merge.

Na segunda alternativa, número não constituiria propriamente um traço associado aos nominais, mas como núcleo funcional apresentaria como requerimento a associação a um $\mathrm{N}$. Assim como acontece no domínio temporal entre $\mathrm{T}$ e $\mathrm{V}$, a relação entre Num e $\mathrm{N}$ poderia ser estabelecida via alçamento de $\mathrm{N}$ para Num ou descida do afixo (contemplando-se possíveis distinções entre as línguas): 
(16)

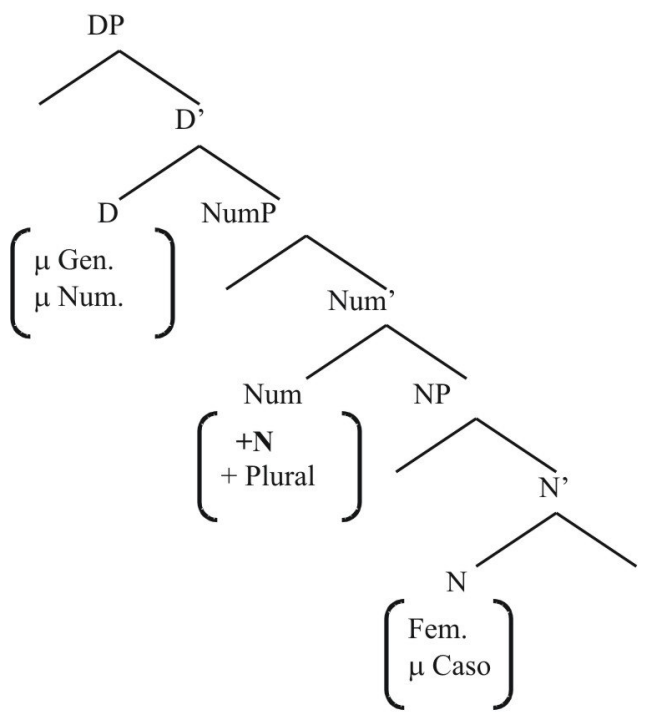

Esses processos, no arcabouço minimalista, têm se valido da noção de traço forte, que força movimento, ou seja, Num, assim como T, apresentaria, a depender da língua, um traço-N forte, demandando movimento, visível em PF. A noção de traço forte foi abandonada em Chomsky (1999) a favor da adoção de EPP, relacionada, no entanto, apenas ao movimento de sintagmas e não de núcleos propriamente. De qualquer maneira, a posição final de conglomerados de núcleos, que diz respeito, em última análise, a questões do parâmetro de ordem das línguas, talvez não precise ser estabelecida na sintaxe. Chomsky (1999, p. 31) especula sobre essa possibilidade ao defender que se trata de questões pertinentes ao componente fonológico: "overt V-to-T raising, T-to- $\mathrm{C}$ raising, and $\mathrm{N}$-to-D raising are phonological properties, conditioned by the phonetically affixal character of the inflectional categories. Considerations of LF uniformity might lead us to suspect that an LF-interpretive process brings together D-N and C-T-V to form word-like LF "supercategories" in all languages, not only those where such processes are visible." 8

A terceira alternativa parece coadunar-se com as assunções mais gerais adotadas no minimalismo: a idéia é manter a noção de que os traços- $\varphi$ são compartilhados pelos elementos presentes no DP. No entanto, número constitui projeção própria e, portanto, a interpretabilidade do traço de 
número está associada a essa projeção. Resta determinar se é possível manter a atuação da operação Agree para dar conta da concordância interna ao DP ou se algum mecanismo adicional se faz necessário. Seria possível estender a análise de Magalhães (2004), com a atuação de Agree, para uma estrutural nominal em que NumP está presente?

Uma primeira questão que se apresenta é o fato de que Agree não poderia ser disparada pelo traço de número em NumP, uma vez que este é interpretável nessa categoria. Haveria a presença de outro traço que levaria à atuação de Agree? Em PB, pode-se admitir a presença de gênero [- interpretável], também associado a NumP (cuja manifestação é visível nos cardinais que se associam a essa projeção: dois, duas):

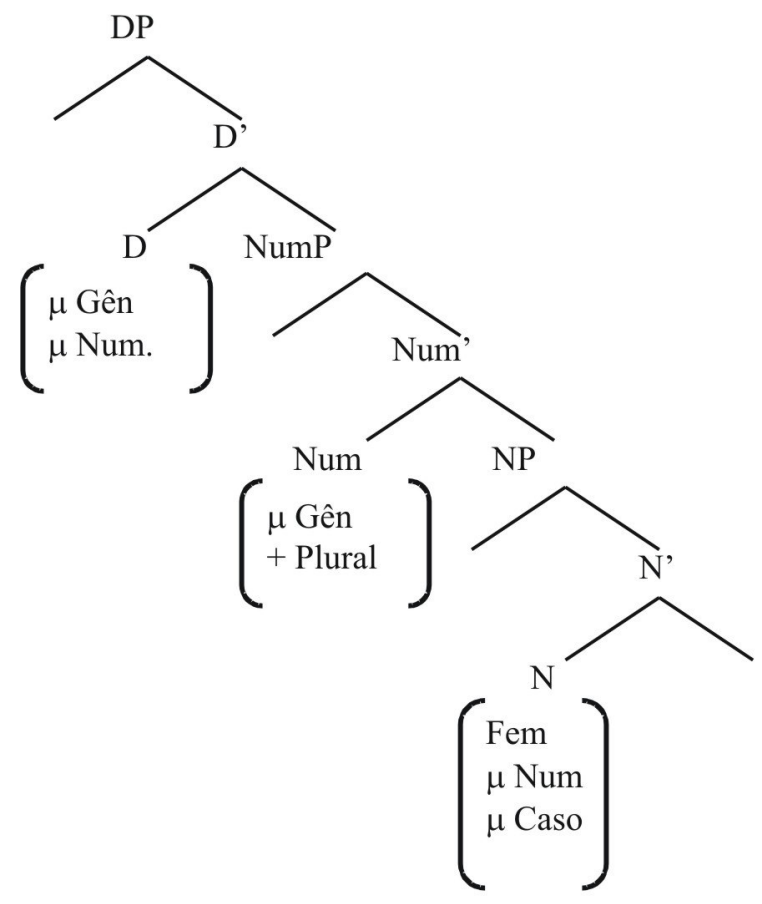

O traço não-interpretável de gênero em NumP dispara o acionamento de Agree. N é encontrado, sendo que o traço de gênero de NumP pode ser valorado, enquanto o traço de número do nome também o será. A valoração dos traços de gênero e número do determinante se dará por meio de um novo 
acionamento da operação Agree, restando o traço de Caso a ser valorado, o que se efetivará pela combinação com uma sonda do tipo $v \mathrm{P}$ ou TP, em cascata, nos moldes de Magalhães (2004), com base em Chomsky (1999). Nesse sentido, como apontado anteriormente, a sonda entraria em relação de concordância com os núcleos presentes na projeção DP, chegando até o $\mathrm{N}$, que mantém o traço de gênero interpretável.

Uma questão que se coloca, portanto, é a da assunção de que apenas conjuntos de traços- $\varphi$ completos seriam capazes de valorar suas contrapartes. Conforme mencionado (ver nota 1), isso parece não poder ser mantido para a concordância interna ao DP. Magalhães (2004) assume que somente traços interpretáveis valorariam suas contrapartes, mas isso não se estenderia para os traços não-interpretáveis já valorados, isto é, o traço de gênero presente em um adjetivo, posicionado entre $\mathrm{D}$ e N, e já valorado por $\mathrm{N}$, não é capaz de valorar o traço não-interpretável de $\mathrm{D}$, que segue sua busca até $\mathrm{N}$, onde esse traço é interpretável. A presença de NumP impõe o mesmo raciocício. Nesse sentido, a concordância interna ao DP seria de natureza levemente distinta da concordância sujeito-verbo ou verbo-objeto. ${ }^{9}$

\section{7- À GUISA DE CONCLUSÃO}

Exploramos aqui a possibilidade de se assumir uma projeção independente relacionada a número no domínio nominal, retomando propostas já adiantadas no arcabouço de GB (RITTER, 1991; PICALLO, 1991), com a preocupação de verificar as implicações que derivariam dessa presença para o mecanismo de concordância interna ao DP, em conformidade com os pressupostos do arcabouço minimalista (particularmente Chomsky, 1999).

Apresentou-se a proposta de Magalhães (2004), em que número, diferentemente da proposta de Chomsky (1995), é tomado como um traço interpretável em D e não em $\mathrm{N}$, e a possibilidade de se estender o mecanismo Agree para a concordância interna ao DP, com base na proposta de Chomsky para as construções participais, é defendida.

A presença da projeção independente NumP, no entanto, demanda repensar a atuação das operações do sistema computacional no que diz respeito à concordância interna ao DP. Na Seção 6, apontou-se a possibilidade de se estender a atuação de Agree, conforme já defendido por Magalhães (2004), para a estrutura nominal em que NumP está presente. 
Apresentamos argumentos a favor de NumP de naturezas distintas (morfológicos, sintáticos, semânticos e formais), consideramos dados de aquisição e elipse no PB, assim como a relevância de propostas acerca de parâmetros relativos a número que fazem referência a essa projeção. Com base nesses argumentos e na discussão relativa ao estabelecimento da concordância interna ao DP, concluímos pela pertinência da hipótese de que NumP constitui uma projeção independente no DP.

\section{NOTAS}

* Este artigo se originou de uma apresentação com o mesmo título no Encontro Nacional do GT de Teoria da Gramática da ANPOLL, realizado em 1 e 2 de dezembro de 2005, sob a forma de pôster. Os autores informam o suporte da FAPERJ (Processo E-26/150.636/2003) ao primeiro autor e do CNPq (Processo 141.323/2005-7) e (Processo 551491/2002-7) aos segundo e terceiro autores respectivamente.

${ }^{1}$ Assumir que traços formais com importe semântico pertinente à referência possam ser considerados núcleos que projetam categorias funcionais independentes apóia uma visão de processamento de base top-down, definindo espaços sintáticos, segundo a qual o acesso a núcleos funcionais na produção de enunciados possibilita tanto a definição dos principais esqueletos sintáticos da sentença, quanto a codificação da referência (Corrêa \& Augusto, 2005; 2006).

${ }^{2}$ O GPPAL - Grupo de Pesquisa em Processamento e Aquisição da Linguagem - está vinculado ao LAPAL - Laboratório de Psicolingüística e Aquisição da Linguagem - ligado ao Programa de Pós-Graduação do Departamento de Letras da PUC-Rio, sendo os dois primeiros autores membros do grupo, sob coordenação do terceiro autor.

${ }^{3}$ Costa e Figueiredo-Silva (2006) remetem a possíveis distinções em relação ao tipo de morfema que a língua privilegia: singleton ou dissociado.

${ }^{4}$ Conforme a análise para os plurais no PB de Wachowicz (2003)

${ }^{5}$ Saab (2005) explora as gradações relacionadas ao gênero. Se gênero semântico (opcional) for distinto de gênero gramatical e também constituir projeção independente, questões morfológicas de boa formação de palavras talvez precisem ser elencadas para se explicar a distinção do contraste nas elipses. Sem dúvida, esse fenômeno merece maior atenção também em relação ao português, mas este não é o foco aqui. 
${ }^{6}$ A proposta de Deprez (2005), em que se assume a camada NumPe um contador (counter), associado a essa projeção, parece coadunar-se bastante bem com a análise de Borer (2005) em que duas projeções relacionadas a número são assumidas: $\mathrm{CL}_{\max } \mathrm{e} * \mathrm{P}$. A camada CLé responsável pela distinção massivos vs. contáveis, enquanto o sistema de contagem - \#P - é o local para a definição específica de quantidades.

A tradução do trecho citado segue:

Parâmetro do Plural

Nas línguas + PL, NumP deve sempre projetar, pelo menos para nomes contáveis - ou seja, mesmo quando o sintagma nominal é singular sem morfologia visível de "plural" ou de "singular" - sendo que essa projeção NumP deve apresentar o contador, i.e., um medidor, que assumo, como primeira aproximação, se traduz pela noção de contagem.

${ }^{7}$ Agradecemos a um dos pareceristas a observação de que há um contraste entre retomada dos singulares bare por pronome lexical (degradada) ou nulo, o que sugere que o pronome nulo deveria ser analisado não como um pro, mas como vestígio de movimento (lateral), nos moldes do proposto por Ferreira (2004) e Rodrigues (2004).

${ }^{8}$ Segue a tradução do trecho citado: "alçamento visível de V-para-T, e alçamento de N-para-D são propriedades fonológicas, condicioinadas pelo caráter foneticamente afixal das categorias flexionais. Considerações acerca da uniformidade em LF nos leva a suspeitar que um processo interpretativo em LF junta D-N e C-T-V para formar 'supercategorias' em todas as línguas, e não somente naqueles em que tais processos são visíveis."

${ }^{9}$ Outro ponto que merece estudo independente e demandaria mais espaço do que o que dispomos nesse momento é a discussão acerca do local de interpretabilidade do traço de pessoa no DP. Para Raposo (1998), com quem concordamos, (ver também Corrêa, 2005) sua associação se dá com D (esse é o local dos pronomes, elementos inerentemente marcados para pessoa). É evidente que essa discussão também se relaciona com a questão levantada acerca da necessidade da presença de um conjunto de traços- $\varphi$ completos para a valoração de contrapartes nãointerpretáveis. 


\section{REFERÊNCIAS}

AUGUSTO, M.R.A. Dados de percepção/compreensão e produção na aquisição: representações gramaticais distintas? Trabalho apresentado no Simpósio: De pronomes a questões teóricas sobre a aquisição gramatical no 7 Encontro Nacional sobre Aquisição de Linguagem, Porto Alegre, 2006.

AUGUSTO, M.R.A. Novos olhares sobre a incongruência entre dados de percepção/compreensão e produção na aquisição: reflexões iniciais. Pôster apresentado no Encontro Nacional do GT de Teoria da Gramática da ANPOLL - 4 e 5/12/2003 - USP. Disponível em: <http://www.letras.puc-rio.br/lapal/ publicacoes.html>

ABNEY, S. The English Noun Phrase in Its Sentential Aspect. 1987. Tese (Doutorado) - MIT.

BENNETT, M. Some extensions of a Montague fragment of English. 1974. Tese (Doutorado) - UCLA.

BOBALJIK, J. Morpho-syntax: the syntax of verbal inflection. 1995. Tese (Doutorado) - MIT, Cambridge Mass.

BORER, H. In Name Only. Structuring Sense, Volume I. Oxford: Oxford University Press. 2005.

BRUGÈ, L. The Positions of demonstratives in the extended nominal projection. In: CINQUE, G. (Ed.). Functional Structure in DP and IP: the cartography of syntactic structures. Oxford University Press, v.1, 2002. p.15-53.

CERQUEIRA, V.C. A sintaxe do possessivo no português brasileiro. 1996. Tese (Doutorado) - IEL/Unicamp.

CHIERCHIA, G. Reference to Kinds across Languages. Natural Language Semantics 6, p. 339-405, 1998.

CHOMSKY, N. The minimalist program, Cambridge, Massachusetts: MIT Press, 1995.

CHOMSKY, N. Minimalist Inquiries: the framework, 1998. Reprinted in: MARTIN, R.; MICHAELS, D.; URIAGEREKA, J. (Ed.). Step by step. Essays on minimalist syntax in honor of Howard Lasnik, Cambridge, Mass. 2000. p. 89-155.

CHOMSKY, N. Derivation by Phase. MITWPL 18. 1999. Reprinted in: KENSTOWICZ, M. (Ed.) Ken Hale. A life in language, Cambridge, Mass., 2001. p. 1-52. 
CINQUE, G. On the evidence for partial N-movement in the Romance DP. In: CINQUE, G.; KOSTER, J.; POLLOCK, J.-Y.; RIZZI, L.; ZANUTTINI, R. (Ed.). Paths towards Universal Grammar. Washington DC:Georgetown University Press, 1994. p. 85-10.

CORRÊA, L.S. Categorias funcionais na integração processador-gramática: pessoa e referência no processamento e na aquisição da linguagem. Projeto apresentado ao CNPq. 2005.

CORRÊA, L. S. Explorando a relação entre língua e cognição na interface: o conceito de interpretabilidade e suas implicações para teorias do processamento e da aquisição da linguagem. Veredas, v.6, n.1, p. 113-129, 2002

CORRÊA, L.M.S.; AUGUSTO, M.R.A. Computação lingüística no processamento on-line: em que medida uma derivação minimalista pode ser incorporada em modelos de processamento? Mesa Inter-GTs no XXI Encontro Nacional da ANPOLL, PUC-SP, São Paulo, 2006.

CORRÊA, L.M.S.; AUGUSTO, M.R.A. Possible loci of SLI from a both linguistic and psycholinguistic perspective. Comunicação apresentada no IX European Group for the Study of Child Language Disorders - EUCLDIS Conference, Paris-Royaumont. 2005.

CORRÊA, L.M.S.; AUGUSTO, M.R.A.; FERRARI-NETO, J. The early processing of number agreement in the DP: evidence from the acquisition of Brazilian Portuguese. In: BAMMAN, D.; MAGNITSKAIA, T.; ZALLER, C. (Ed.). A Supplement to the Proceedings of the $30^{\text {th }}$ Annual Boston University Conference on Language Development, 2006.

CORRÊA, L.M.S., AUGUSTO, M.R.A.; FERRARI-NETO, J. The early processing of number agreement in the DP: evidence from the acquisition of Brazilian Portuguese. Trabalho apresentado no Boston University Conference on Language Development. 2005.

CORRÊA, L.M.S., AUGUSTO, M.R.A., FERRARI-NETO, J.; NAME, M.C.L. Similarities and differences in the acquisition of number and gender: an experimental investigation on the basis of Brazilian Portuguese. Trabalho apresentado no Second Lisbon Meeting on Language Acquisition. 2004.

COSTA, J.; FIGUEIREDO-SILVA, M.C. Nominal and verbal agreement in Portuguese: an argument for Distributed Morphology. In: FIGUEIREDO SILVA, M.C.; COSTA, J. (Org.). Studies on Agreement. Amsterdam: John Benjamins Publishing Company, 2006. 
DEPREZ, V. Morphological number, semantic number and bare nouns. Lingua 115, p. 857-883, 2005.

DRYER, M. S. Plural words. Linguistics 27, p. 865-895, 1989.

FERRARI-NETO, J. Reconhecimento do número gramatical e processamento da concordância de número no sintagma determinante na aquisição do português brasileiro. 2003. Dissertação (Mestrado) - PUC-Rio, Rio de Janeiro.

FERRARI-NETO, J., CORRÊA, L.S.; AUGUSTO, M.R.A. O Processamento da Informação de Interface na Aquisição do Sistema de Número Gramatical no DP em Português Brasileiro. Atas do IV Congresso Internacional da ABRALIN, p. 1075-1082, 2005.

FERREIRA, M. B. Hyperraising and Null Subjects in Brazilian Portuguese. In: CASTRO, Ana; HACQUARD, Valentine; SALANOVA, Andres; FERREIRA, Marcelo (Org.). Romance. Collected Papers on Romance Syntax. Cambridge: MITWPL, 2004. p. 57-85.

GIUSTI, G. The categorial status of quantified nominals, Linguistische Berichte 136, p. 438-454, 1991.

LONGOBARDI, G. Proper names and the theory of N-movement in syntax and logical form. Linguistic Inquiry 25, 1994.

LOPES, R. Estágios no processo de aquisição de número no DP do português brasileiro. Letras de Hoje, Porto Alegre, v. 39, n.3, p. 157-171, 2004.

MAGALHÃES, T. A valoração de traços de concordância dentro do DP. D.E.L.T.A. 20: 1, p. 149-170, 2004.

MASULLO, P.; DEPIANTE, M. Gender is in the lexicon, number is in the syntax: evidence from nominal ellipsis in Spanish. GLOW 27, 2004.

MÜLLER, A. Genericity and the Denotation of Common Nouns in Brazilian Portuguese. D.E.L.T.A. 18.2. São Paulo: PUCSP, p. 287-308, 2002.

MUNN, A.; SCHMITT, C. Bare Nominals and the Morpho-syntax of Number, In: CRESTI, D.; SATTERFIELD, T.; TORTORA, C. (Ed.). Current Issues in Romance Linguistics: Selected Papers from the XXIXth Linguistic Symposium on Romance Languages (LSRL), Ann Arbor, April 1999. Amsterdam: John Benjamins, p. 217-231, 2001.

MUNN, A.; SCHMITT, C. Number and indefinites. Lingua 115, p. 821-855, 2005. 
OLSEN, S. AGR(eement) in the German Noun Phrase. In: BHAYY, C.H.R.; LÖBEL, E.; SCHMITT, C. (Ed.). Syntactic phrase structure phenomena in noun phrase and sentences. Amsterdam:John Benjamins, p. 39-49, 1989.

PICALLO, C. Nominals and nominalization in Catalan. Probus 3 (3), p. 279316, 1991.

POLLOCK, J.-Y. Verb movement, Universal Grammar and the structure of IP. Linguistic Inquiry 20, p. 365-424, 1989.

RAPOSO, E. Some observation on the pronominal system of Portuguese. CatWPL 6, p. 59-93, 1998.

RITTER, E. Evidence for number as a nominal head. In: GLOW Newletter. Paper presented at GLOW-14, 1991.

RODRIGUES, C. Super-raising in Partial Pro-drop Languages. In: BOECKX, Cedric (Org.). Agreement.: Oxford University Press, 2004.

SAAB, A. On morphological sloppy identity in Spanish nominal ellipsis. Ms. Universidad Nacional del Comahue/CONICET, Argentina. 2005.

SAUERLAND, U. A New Semantics for Number. In: YOUNG, R.; ZHOU, Y. (Ed.). Proceedings of SALT 13, CLC Publications, Cornell University, Ithaca, N.Y. 2003.

SCHMITT, C.; MUNN, A. Bare nominals, morphosyntax, and the nominal mapping parameter. Michigan State University. 2000. Disponível em: <overcite.lcs.mit.edu/schmitt00 bare.html.>

SHLONSKY, U. Quantifiers as functional heads: a study of quantifier float in Hebrew, Lingua 84, p. 159-180, 1991.

VANGSNES, O.A. On noun phrase architecture, referentiality, and article systems. Studia Linguistica 55(3): p. 249-299, 2001.

WACHOWICZ, T.C. A semântica dos reticulados para os plurais em PB. In: MULlER, A.; NEGRÃO, E.V.; FOLTRAN, M.J. (Org.). Semântica Formal. São Paulo: Editora Contexto, 2003.

ZOCCA, C. O que não está lá? Um estudo sobre morfologia flexional em elipses. 2003. Dissertação (Mestrado) - Unicamp, São Paulo. 